Nervenarzt 2016 $87: 150-160$

DOI 10.1007/s00115-015-0066-9

Online publiziert: 26. Januar 2016

(c) The Author(s) 2016. This article is available at SpringerLink with Open Access

CrossMark

J. de Jonge' $\cdot$ M. Kalisvaart ${ }^{1} \cdot$ M. van der Hoeven' ${ }^{1}$ J. Epker ${ }^{2}$. J. de Haan ${ }^{2}$.

J. N. M. IJzermans' ${ }^{1}$ F. Grüne ${ }^{3}$

'Department of Surgery, Erasmus University Medical Centre, Rotterdam, Netherlands

${ }^{2}$ Department of Intensive Care Medicine, Erasmus University Medical Centre, Rotterdam, Netherlands

${ }^{3}$ Department of Anaesthesiology, Erasmus University Medical Centre, Rotterdam, Netherlands

\title{
Organspende nach Herz- und Kreislauftod
}

Herz- oder Kreislauffunktion den kritischen Rand zwischen Leben und Tod definiert. Das Gehirn erbringt die für den gesamten Menschen notwendige Integrationsleistung, ohne die er nicht als organismische, mentale und seelische Ganzheit existieren könnte [4, 11, 24-26].

$\mathrm{Zu}$ Beginn der 1950er Jahre wurden Fälle eines „coma dépassé“ (irreversibles Koma) beschrieben. Es handelte sich hierbei um Patienten mit schwerer irreversibler Hirnschädigung, bei denen Atmung und Herz-Kreislauf-Tätigkeit künstlich stimuliert wurden, ohne dass die Hoffnung bestand, dass sich deren Gehirnfunktionen jemals wiederherstellen würden. Eine Kommission der Harvard Medical School veröffentlichte im Jahre 1968 eine genaue Definition der irreversibel erloschenen Gesamtfunktion des Gehirns und schlug vor, den entsprechenden Zustand als Hirntod zu bezeichnen, als sicheres Todeszeichen anzuerkennen und als Todeskriterium festzulegen [2]. Begründet wurde dies damit, sowohl den Status der komatösen Patienten zu klären und die künstliche Beatmung einstellen zu können, als auch Kontroversen bei der Beschaffung von Organen zur Transplantation zu vermeiden.

In den USA wurde erst 1981 von der „National Conference of Commissioners on Uniform State Law" ein Konzept einer gesetzlichen Todesdefinition vorgeschlagen, die neben dem „klassischen“ HerzKreislauf-Tod ausdrücklich den Gesamthirntod als Tod des Menschen umfasste. Als dessen Kennzeichen wurden festgelegt:
1. keine Rezeptivität und Reaktivität,

2. keine spontanen Bewegungen und Atmung,

3. keine Reflexe und

4. flaches Elektroenzephalogramm (EEG).

Dieses Konzept wurde 2008 durch das President's Council on Bioethics unter dem Titel „Controversies in the Determination of Death“ überarbeitet, da der anhaltende Dissens zum Hirntodkriterium sowie neue empirische Ergebnisse zum integrierten Funktionieren des Körpers von Hirntoten eine erneute Debatte über den Hirntod erforderten [20]. Viele nationale und internationale Rechtsordnungen hatten gleichfalls den Gesamthirntod als Tod des Menschen ausdrücklich oder stillschweigend akzeptiert und als hinreichende Voraussetzung für die postmortale Organspende implementiert. International gibt es allerdings erhebliche Unterschiede in den gesetzlichen Regelungen und Normierungen bezüglich Definition, Kriterien und Zeitpunkt des Todes [22, 26].

Grundsätzlich dürfen Organe nur Toten entnommen werden („dead donor rule“) [31]. Für die meisten Rechtsordnungen in Europa ist die Organspende nach Gesamthirntod legal. Der mentale Tod ist auch für die meisten Kritiker des Hirntodkonzepts eine Bedingung für den Tod des Menschen. Grundsätzliche Bewertungsunterschiede bestehen aber in der Frage, ob ein „hirntoter“ Mensch auch in organismischer Hinsicht schon als tot zu bezeichnen ist. Der Anschein von Lebendigkeit hirntoter Pati- 
enten ist vor allem für deren Angehörige ein Dilemma. Atembewegungen, rosige Hautfarbe, Bartwuchs etc. werden allgemein mit dem Leben verbunden, auch wenn dies nur künstlich durch die Intensivtherapie erreicht wird. Bis zu einer Organentnahme werden hirntote Patienten wie alle anderen Intensivpatienten versorgt, bis hin zur ausdrücklichen Ansprache durch Pflegekräfte und Ärzte, was nicht dem Umgang mit „echten“ Leichen entspricht [17]. Die Amerikanische Fachgesellschaft für Neurologie hat 2010 der bislang vorgeschriebenen Hirntoddiagnostik eine fehlende wissenschaftliche Fundierung bescheinigt [35]. Der Einsatz von Analgetika, Anästhetika und Muskelrelaxanzien bei der Organentnahme werden daher von einigen wissenschaftlichen Arbeitsgruppen und Rechtsordnungen (z. B. Schweiz) als notwendig angesehen, da bei hirntoten $\mathrm{Pa}$ tienten mitunter noch höhere Hirn- und Rückenmarksfunktionen feststellbar seien und dadurch unerwünschte Veränderungen des Blutflusses während der Explantation auftreten können [10, 36].

\section{》) In den meisten Ländern Europas ist die Organspende nach Gesamthirntod legal}

Aufgrund des Zweifels, dass sich die Gleichsetzung von Tod und Hirntod auch naturwissenschaftlich nicht aufrechterhalten lässt, werden in der aktuellen Diskussion über den Hirntod vor allem drei Konsequenzen diskutiert:

1. eine neue Rechtfertigung der Gleichsetzung von Hirntod und Tod,

2. die Abschaffung der Tote-SpenderRegel und

3. der Verzicht auf Organentnahmen aus hirntoten Patienten.

Die dritte Option kommt für die meisten Staaten nicht infrage, da sie Qualität und Quantität des Organangebots reduziert würde. Derzeit wird von den meisten nationalen Ethikräten eine neue Rechtfertigung der Gleichsetzung von Hirntod und Tod oder besser eine Neudefinition von Leben und Tod verfolgt. Diese orientiert sich besonders an der Selbsterhaltung des Menschen durch Auseinan- dersetzung mit der Umwelt und weicht somit vom Lebensbegriff der Biologie ab. Dieser Ansatz erweckt jedoch bei den meisten Kritikern den Eindruck einer interessengeleiteten Ethik, da diese philosophische Neudefinition des Hirntods als Zugeständnis an das Tötungsverbot und die Transplantationsmedizin interpretiert werden kann [17, 30-32].

Aus klinischer Sicht ist der Nichtidentität von Hirntod und Tod zu widersprechen [16]. Eine lege artis durchgeführte Hirntoddiagnostik gewährleistet die Sicherheit der korrekten Feststellung der irreversibel erloschenen Gesamtfunktion des Großhirns, des Kleinhirns und des Hirnstamms. Sie identifiziert aber auch Patienten, bei denen eben nicht ein kompletter Verlust der Hirnaktivität vorliegt. Bislang ist kein Fall bekannt, bei dem ein Patient unrichtigerweise für hirntot erklärt oder eine Organentnahme erfolgte, nachdem eine komplette, formal korrekte Hirntoddiagnostik durchgeführt wurde. Weltweit hat sich die Auffassung vom Hirntod als dem Tod des Menschen weitgehend durchgesetzt. Darüber hinaus ist zu bemerken, dass solche Überlegungen nur wenig dazu beitragen können, die Verunsicherung und Desorientierung weiter Teile der Bevölkerung in der Frage des Hirntods abzubauen.

\section{》) Entscheidend ist der Grad des gesellschaftlich-juristischen Konsenses}

Die Übergänge beim Sterbeprozess wie bei der Geburt bleiben fließend und sind schwierig zu definieren. Viele Bioethiker bezeichnen daher die Gleichsetzung von Tod und Hirntod als „legale Fiktion“. Sie plädieren für die zweite Option, also die Abschaffung der „dead donor rule“ [4, 11]. Die Frage, ob eine Beendigung des Tötungsverbots bei ausdrücklicher Zustimmung des Patienten ethisch akzeptabel und rechtlich möglich ist, bleibt schwierig zu beantworten. Mit der Feststellung des Hirntodes und/oder bei infauster Prognose entfällt die Pflicht und das Recht des Arztes, eine kurativ ausgerichtete Therapie fortzusetzen. Stattdessen steht nun der Wille des betroffenen Patienten im Vordergrund. Die Entschei- dung, sein Leben nicht nur durch die Beendigung lebenserhaltender Maßnahmen, sondern mit einem Akt der Organspende zu beenden, sollte respektiert werden (Recht auf Selbstbestimmung). Organexplantationen, die auf ausdrücklichen Wunsch des sterbenden Patienten geschehen, könnten dann als eine besondere Form des Sterbenlassens („allowing to die") bewertet werden [30]. Dann wären Ärzte von der Befürchtung entlastet, bei der Organentnahme einen Menschen zu töten.

Das moralisch-ethische Dilemma in der Debatte über den Hirntod und Organtransplantation jedoch bleibt: Die Explantation möglichst vitaler Organe zur Rettung des Empfängers bei gleichzeitig würdevollem Sterben des Organspenders lässt sich vielleicht nie für alle Beteiligten befriedigend lösen. Entscheidend ist hier der Grad des gesellschaftlich-juristischen Konsenses bzw. der möglichst weit voran getriebene medizinische-ethische Diskurs, ob jemand tot ist, oder vielmehr, ob er als tot gilt [23].

\section{Organspende in den Niederlanden: „donation after circulatory death"}

In den Niederlanden werden jährlich ca. 76.000 Patienten intensivmedizinisch behandelt. Ungefähr $15 \%$ dieser Patienten befinden sich am Lebensende und versterben. Ein Fortsetzen der kurativen Therapiemaßnahmen in dieser Situation wird in den Niederlanden als „sinnlose medizinische Behandlung“ verstanden. Der Übergang von einer kurativen zur palliativen Therapie oder "end of life care“ ist in den Niederlanden schon seit einigen Jahren gesetzlich und durch Leitlinien geregelt [9]. Von diesen ca. 8500 palliativ behandelten Patienten auf Intensivstationen, versterben mehr als $80 \%$ nach Beenden der lebenserhaltenden Therapie.

Ein Teil dieser Patienten, die den „point of no return“ vom Leben zum Sterben überschritten haben, sind potenzielle Organspender. Unter Berücksichtigung der gesetzlichen Rahmenbedingungen kann eine postmortale Organspende sowohl bei spendenden Personen nach „primärem Hirntod“ infolge einer direkten Schädigung des Gehirns („donation 
after brain death“, DBD) als auch nach „sekundärem Hirntod“ infolge eines irreversiblem Herz-Kreislauf-Stillstands durchgeführt werden. Man spricht dann von "non heart-beating donors“ (NHBD) oder auch von ,donation after circulatory death“ (DCD). Hierbei wird eine zeitliche Komponente miteinbezogen. Bei plötzlichem Herzstillstand eines potenziellen Organspenders in oder außerhalb des Krankenhauses tritt der Tod unerwartet ein. Bei Organspendern, deren lebenserhaltenden Maßnahmen beendet werden (wie z. B. auf der Intensivstation), wird der Tod hingegen erwartet. Dies führt zu einer weiteren Unterscheidung zwischen erwarteter und unerwarteter Organspende nach Herz-Kreislauf-Tod (expected, eDCD; unexpected, uDCD, - Tab. 1; [14, 15, 22]).

Das Konzept einer Organentnahme bei Spendern mit irreversiblem HerzKreislauf-Stillstand ist nicht neu. In den 1970er Jahren wurden die ersten transplantierten Nieren-NHB-Spendern entnommen. Das erste Herz, das von Christiaan Barnard transplantiert wurde, stammte gleichfalls von einem NHBSpender. Die Praxis der DCD wurde jedoch damals aufgegeben aufgrund der im Vergleich zur DBD-Transplantation schlechteren Resultate.

Das größte Problem in den Niederlanden war die äußerst geringe Anzahl an potenziellen Organspendern. Sehr alte Patienten und Patienten mit z. B. Tumorerkrankungen kamen als potenzielle Organspender nicht mehr infrage. Bei Patienten ohne Organspenderausweis oder Registrierung als Organspender stimmten die Angehörigen nur zu einem Drittel einer Organspende zu. Die Spenderzahl in den Niederlanden war 1999 mit 10,4 Transplantationen pro 1 Mio. Einwohner die niedrigste in ganz Europa und das sind deutlich weniger als das weltweit definierte Potenzial von 40 pro 1 Mio. Einwohner (• Abb. 1; $[6,18])$. Die Wartezeit auf ein Spenderorgan war lang. Patienten auf der Transplantationswarteliste mit terminalen Nierenversagen und Hämodialysetherapie hatten damals eine statistische Überlebenschance von weniger als $50 \%$, also weitaus schlechter im Vergleich zu Patienten mit malignen Tumorerkrankungen. Bis zu einem

Nervenarzt 2016 $\cdot 87: 150-160 \quad$ DOI 10.1007/s00115-015-0066-9

(c) The Author(s) 2016

J. de Jonge - M. Kalisvaart · M. van der Hoeven · J. Epker · J. de Haan · J. N. M. IJzermans . F. Grüne

\section{Organspende nach Herz- und Kreislauftod}

\section{Zusammenfassung}

In den Niederlanden leben ca. 17 Mio. Einwohner. Die Anzahl an potenziellen postmortalen Organspender war 1999 mit 10 Spenden pro 1 Mio. Einwohner die niedrigste in ganz Europa. Medizinische Fachgesellschaften, öffentliches Gesundheitswesen, Krankenkassen und Gesetzgeber mussten gemeinsam Wege zu einer verbesserten Organallokation, Logistik und Anzahl von Transplantationen finden. Nach andauernder Debatte zu medizinischethischen Themen der Organtransplantation, konnten sich alle Beteiligten schrittweise auf ein gesellschaftlich-medizinischjuristisches Regelwerk zur Organspende und Transplantation einigen. Neben Verbesserung der Abläufe einer Organspende nach Hirntod ("donation after brain death“, DBD) war der wichtigste Schritt die Einführung der Organspende nach Herz-Kreislauf-Tod („donation after circulatory death", DCD). Durch Maßnahmen wie z. B. die Einführung einer nationalen Organspenderdatenbank, verbesserte Aufklärung der Öffentlichkeit,
Weiterbildung auf den Intensivstationen, Leitlinien zur intensivmedizinischen Behandlung am Lebensende, Einsatz eines Transplantationskoordinators vor Ort, Einführung autonomer Explantationsteams und strikte Verfahrensanweisungen zum Ablauf der Organspende konnten viele praktische Fragen zu Logistik und Aufgabenteilung der DBD und DCD beantwortet werden.

Die Anzahl an postmortalen Organspenden stieg 2014 auf 16,4 pro 1 Mio. Einwohner. Mittlerweile stammen bis zu $60 \%$ der Organspenden in den Niederlanden aus einem DCD-Verfahren, verglichen mit ungefähr $10 \%$ in den USA.

Dieser Übersichtsartikel beschreibt die Entwicklungen und Abläufe einer postmortalen Organspende in den Niederlanden nach 15 Jahren Erfahrung mit DCD.

\section{Schlüsselwörter}

Organtransplantation - Medizinethik . Hirntod - Versorgung am Lebensende . Niederlande

\section{Organ donation after circulatory death}

\section{Abstract}

Approximately 17 million inhabitants live in the Netherlands. The number of potential organ donors in 1999 was the lowest in Europe with only 10 donors per million inhabitants. Medical associations, public health services, health insurance companies and the government had to find common solutions in order to improve organ allocation, logistics of donations and to increase the number of transplantations. After a prolonged debate on medical ethical issues of organ transplantation, all participants were able to agree on socio-medico-legal regulations for organ donation and transplantation. In addition to improving the procedure for organ donation after brain death (DBD) the most important step was the introduction of organ donation after circulatory death (DCD). Measures such as the introduction of a national organ donor database, improved information to the public, further education on intensive care units (ICU), guidelines for end of life care on the ICU, establishment of transplantation coordinators on site, introduction of autonomous explantation teams and strict procedures on the course of organ donations, answered many practical issues about logistics and responsibilities for DBD and DCD. In 2014 the number of postmortem organ donations rose to 16.4 per million inhabitants. Meanwhile, up to $60 \%$ of organ donations in the Netherlands originate from a DCD procedure compared to approximately $10 \%$ in the USA. This overview article discusses the developments and processes of deceased donation in the Netherlands after 15 years of experience with DCD.

\section{Keywords}

Organ transplantation - Medical ethics · Brain death . End of life care $\cdot$ Netherlands 
Tab. 1 Maastricht-Kriterien zur Organspende nach Herz-Kreislauf-Stillstand („donation after circulatory death")

\begin{tabular}{|llll}
\hline Kategorie & Definition & $\begin{array}{l}\text { Lokalisation der } \\
\text { DCD }\end{array}$ & Anmerkungen \\
\hline I & $\begin{array}{l}\text { Herz-Kreislauf-Stillstand bei } \\
\text { Klinikankunft }\end{array}$ & Notaufnahme & $\begin{array}{l}\text { Unkontrolliert/ } \\
\text { unerwartet }\end{array}$ \\
\hline II & $\begin{array}{l}\text { Herz-Kreislauf-Stillstand nach } \\
\text { erfolgloser Reanimation }\end{array}$ & Notaufnahme & Unkontrolliert/ \\
& $\begin{array}{l}\text { Herz-Kreislauf-Stillstand nach } \\
\text { Abbruch lebenserhaltender } \\
\text { III }\end{array}$ & Intensivstation & Kontrolliert/erwartet \\
& $\begin{array}{l}\text { Herzstillstand bei Hirnstamm- } \\
\text { tod }\end{array}$ & Intensivstation & Kontrolliert/erwartet \\
\hline IV & $\begin{array}{l}\text { Herzstillstand während des } \\
\text { Klinikaufenthalts }\end{array}$ & $\begin{array}{l}\text { Normalstation/ } \\
\text { Intensivstation }\end{array}$ & Unkontrolliert/ \\
\hline V & unerwartet \\
\hline a Die Kategorie V wurde erst 2000 hinzugefügt [14, 37]. & \\
DCD „donation after circulatory death" & & \\
\hline
\end{tabular}

Drittel der Patienten auf der Warteliste für Lebertransplantationen erlebten ihre Transplantation nicht mehr $[11,18]$. Darüber hinaus stieg die Nachfrage an Spenderorganen.

In den Niederlanden, aber auch in anderen Ländern, wie z. B. in Belgien, Lettland und Großbritannien, wurde die Entnahme von Organen bei Spendern mit irreversiblem Herzstillstand in den letzten Jahren wieder in Betracht gezogen [15]. Eine Maastrichter Arbeitsgruppe untersuchte schon seit 1982 die Möglichkeit der Wiedereinführung des DCD-Konzeptes. Potenzielle NHBOrganspender werden nach den sog. Maastricht-Kriterien in 4 verschiedene Kategorien klassifiziert (• Tab. 1). Seit 1989 wurden in einem Pilotprojekt Organspender aus der Kategorie II (Spender nach unerwartetem HerzKreislauf-Stillstand und erfolgloser Reanimation) rekrutiert. Seit 1998 wurde das Forschungsprogramm durch Organspender der Kategorie III (Spender mit infauster Prognose, Behandlungsabbruch, kontrollierter oder erwarteter Herz-Kreislauf-Stillstand) erweitert. Diese Organspender eigneten sich dann für die DCD-Multiorganspende. Die qualitativen Mängel der DCD-Organe konnten durch eine verbesserte Behandlung der potenziellen Spender, effektivere Logistik und standardisiertes Vorgehen der Explanation mittlerweile erheblich verbessert werden $[11,22]$.
Das Maastrichter DCD-Programm wurde kurze Zeit später landesweit in den Niederlanden eingeführt (Dutch Organ Donation Act). Die Organspender der Kategorie III (DCD) wurden der Gruppe IV (Hirntod, DBD) gesetzlich gleichgesetzt. Dennoch blieb die Bilanz enttäuschend. Im Jahr 2008 leitete die niederländische Regierung einen Aktionsplan für Organspende ein (Masterplan Organdonatie), die zu einer Zunahme der Transplantationen führte [18].

Außerhalb Europas haben nur Japan, Australien und die Vereinigten Staaten langjährige Erfahrungen mit der DCD. Der Hirntod als Todeskriterium für die Organspende wird in Japan aus kulturellen Gründen kaum akzeptiert. Hier findet die postmortale Organspende ausschließlich nach DCD statt. In Kanada, Neuseeland und China wurden vor kurzem DCD-Programme eingeführt [3, 5, $15]$.

Die Ergebnisqualität der ersten Projekte zur Nierentransplantation nach DCD war gut. Unterschiede gab es in der frühen postoperativen Phase: Patienten nach DCD-Nierentransplantation zeigten eine höhere Inzidenz von verzögerter Funktion („delayed graft function“, DGF; [29, 33, 34]). Die Langzeitfunktion der transplantierten DBD-Nieren war $10 \%$ schlechter, verglichen mit Zahlen nach DCD (• Abb. 2a). Ursachen hierfür sind wahrscheinlich in der an- fänglich schlechteren Organqualität und Selektion der Empfänger zu finden.

Bei Patienten nach DCD-Lebertransplantation zeigten sich hingegen etwas schlechtere Ergebnisse verglichen mit Zahlen nach DBD. Grund hierfür war eine $40 \%$ höhere Inzidenz an Komplikationen des biliären Systems und der ischämischen Cholangiopathie (- Abb. 2b; [1, 21, 28]). Der Einsatz maschineller Organkonservierung hat positive Effekte auf die Qualität der Spenderorgane. In klinischen Studien wird derzeit geprüft, ob es zu einer Abnahme der verzögerten Transplantatfunktion kommt und das Langzeitüberleben der Empfänger verbessert werden kann [12].

\section{Entwicklung der postmortalen Organspenden in den Niederlanden}

Das Zusammenspiel mehrerer Faktoren begünstigte die Zunahme der DCD in den Niederlanden. Die Anzahl der Patienten, die nach Beenden der lebenserhaltenden Maßnahmen an einem Herz-Kreislauf-Stillstand (sekundärer Hirntod) starben, waren im Vergleich zur Anzahl der verstorben Patienten nach primärem Hirntod deutlich höher. So hatte die Anzahl der Organspender nach primärem Hirntod und DBD von 1996 bis 2013 um $50 \%$ abgenommen. In dieser Zeitspanne konnte eine deutliche Reduktion von schwerstverletzten Unfallopfern beobachtet werden. Die Verkehrssicherheit hatte sich deutlich verbessert.

Mittlerweile ist der Anteil der postmortalen Organspender nach Verkehrsunfall auf $20 \%$ gesunken.

Etwa $60 \%$ der Transplantationen stammen von Spendern mit neurovaskulären Erkrankungen. Durch Prävention, neuere neurochirurgische Techniken, interventionelle radiologische Techniken und die moderne Intensivmedizin sank aber auch die Mortalität und Morbidität der Patienten mit neurovaskulären Erkrankungen. Dadurch begünstigt nahm das Durchschnittsalter eines niederländischen Organspenders in den letzten 15 Jahren um 10 Jahre zu $[11,18]$. 


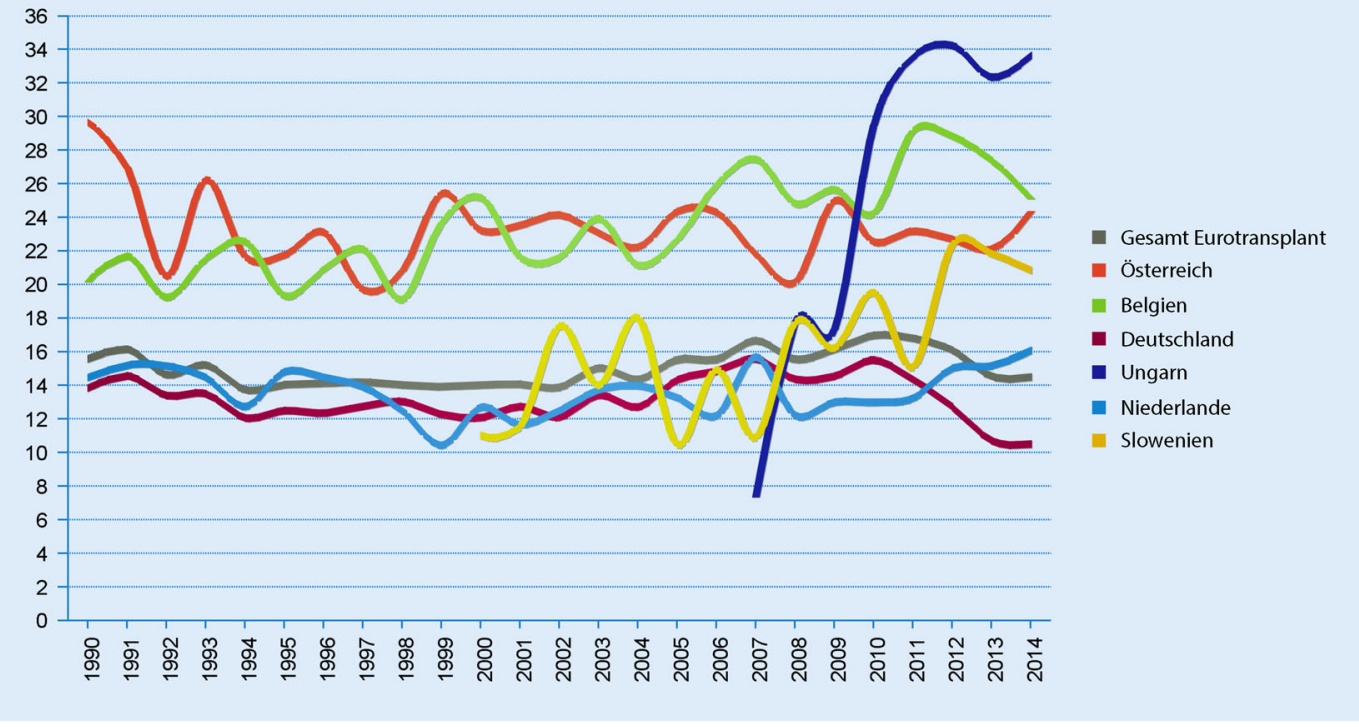

Abb. $1 \varangle$ Postmortale Organspenden pro 1 Mio. Einwohner, Jahr und Land. (Eurotransplant Database [38]), Manara et al. [15]
Die Anzahl von Personen mit Organspenderausweis oder Organspenderregistrierung war auch in den Niederlanden lange rückläufig. Viele Angehörige potenzieller Organspender verweigerten ihre Zustimmung zur Organspende. Hier gab es für die Öffentlichkeit einen erheblichen Bedarf, um Missverständnisse bezüglich der Definition des Todes auszuräumen. Seit 1998 gibt es in den Niederlanden ein öffentliches Organspenderegister. Jährlich ruft die niederländische Regierung ihre Mitbewohner auf, sich registrieren zu lassen („donorweek",https://wordookdonor.nl). Bislang sind $42 \%$ aller Niederländer registriert, $26 \%$ stimmen einer Organspende zu und ca. $16 \%$ lehnen diese ab [18].

Der Übergang von kurativer zu palliativer Therapieform bei schwerkranken Patienten und die Betreuung der Angehörigen stellt das Behandelteam einer Intensivstation fortwährend vor Herausforderungen. Der Abbruch lebenserhaltender Maßnahmen, „end of life care" und die Frage nach Organspende wurden für Intensivmediziner oft als Dilemma erfahren. So waren Anmeldung und Organisation eines potenziellen Organspenders innerhalb der niederländischen Intensivstationen sehr unterschiedlich geregelt. Leitlinien und gesetzliche Rahmenbedingungen zur palliativen Therapie auf der Intensivstation und Aufgabenteilteilung in der Organallokation konnten hier
Verbesserungen erwirken $[9,11,18]$. Die Art der Organspende, DBD oder DCD, spielten in diesem Kontext eher eine untergeordnete Rolle.

Von 2000 bis 2014 nahm die Zahl der Transplantationen in den Niederlanden deutlich zu. Bei der DCD wurden im Durchschnitt zwei Organe entnommen, bei der DBD waren es 3,5 Organe. Mittlerweile stammen $60 \%$ aller transplantierten Organe von DCD (• Abb. 3). In anderen Ländern, wurde eine ähnliche Zunahme von DCD beobachtet; in Belgien waren es ca. $20 \%$, in Großbritannien ungefähr $35 \%$ und in den USA ca. $10 \%[5,18]$.

\section{Ablauf einer Organspende (DCD) in den Niederlanden}

In den letzten 15 Jahren wurden in den Niederlanden viele nationale und lokale Projekte zur Verbesserung des DCDProzesses durchgeführt [18]. Die Konsequenzen einzelner Projekt war oft nicht vorauszusagen. Viele Erfahrungen wurden durch Versuch und Irrtum gemacht.

Neben der andauernden Debatte zu medizinisch-ethischen Themen und der Festlegung auf ein gesellschaftlich-juristisches Regelwerk mussten viele praktische Fragen zu Logistik und Aufgabenteilung der DBD und DCD beantwortet werden.

\section{Organspende und das intensivmedizinische Team}

Die Entscheidung über den Wechsel von einer kurativen zur palliativen Therapie und das Beenden lebenserhaltender Maßnahmen muss in den Niederlanden entsprechend den nationalen Leitlinien durch ein multidisziplinäres Team erfolgen [9]. Haltung und Wissensstand zum Organspendeprozess unterschieden sich zwischen Teams von Transplantationszentren im Vergleich zu Nichttransplantationszentren. Pflegekräfte und Ärzte von Nichttransplantationszentren sind ausschließlich mit der Betreuung potenzieller Organspender und ihrer Angehörigen beauftragt. Diese Aufgabe ist oft emotional sehr belastend und kann zu einer negativen Haltung hinsichtlich der Organspende führen. In den Transplantationszentren erleben Pflegekräfte und Ärzte gleichzeitig auch die positiven Patientenverläufe in der Transplantationsmedizin.

Weiterhin gibt es Unterschiede in der Haltung zu DBD vs. DCD. In einer britischen Umfrage zur Organspende war die Anzahl der positiven und negativen Begriffe, die mit der DBD und DCD assoziiert waren, signifikant unterschiedlich (DBD $=68$ Wörter positive, 25 negative; DCD $=29$ Wörter positive, 89 negative; [7]). Die kontinuierliche Weiterbildung des intensivmedizinischen Behan- 

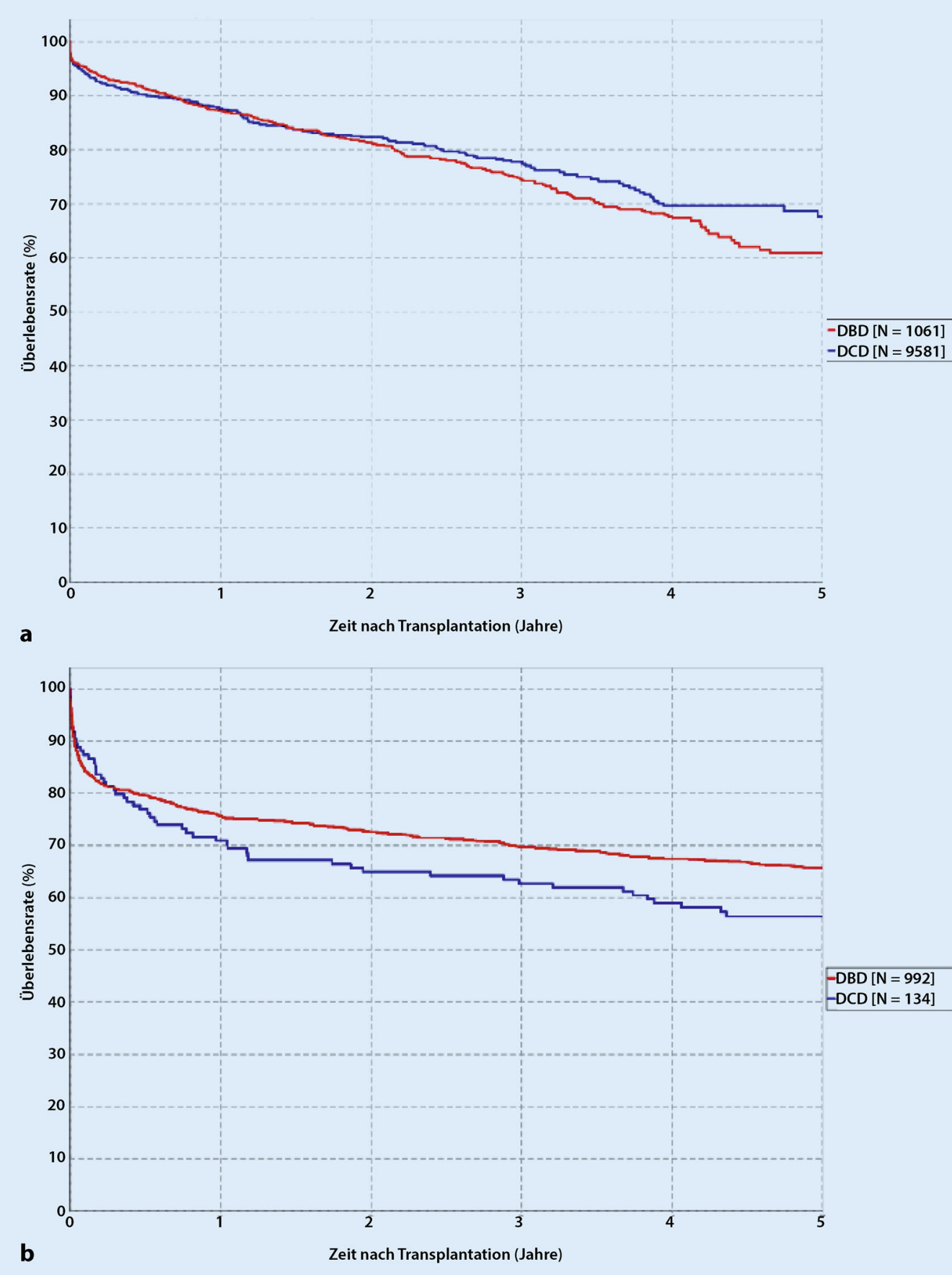

Abb. $2<$ a 5-JahresÜberlebensrate nach Nierentransplantation (Daten aus 2009-2013). Die Langzeitfunktion der transplantierten DBD-Nieren war $10 \%$ schlechter, verglichen mit Zahlen nach DCD. Ursachen hierfür sind wahrscheinlich in der anfänglich schlechteren Organqualität und Selektion der Empfänger zu finden. b 5-Jahres-Überlebensrate nach Lebertransplantation (Daten aus 2000-2009). Die Langzeitergebnisse bei Patienten nach DCD-Lebertransplantation sind etwas schlechter, verglichen mit Zahlen nach DBD. Nach DCD zeigte sich eine $40 \%$ höhere Inzidenz an Komplikationen des biliären Systems und der ischämischen Cholangiopathie $[1,21,28]$. Rote Linie DBD (Donation after Brain Death $=$ heart-beating donation, Organspende nach Hirntod = Organspende bei Herzschlag), Blaue Linie DCD (Donation after Circulatory Death $=$ non-heart-beating donation, Organspende nach Herz-Kreislauf-Tod = Organspende ohne Herzschlag). (Quelle: [19])

delteams zu Themen der „end of life care“, Organspende und Transplantationsmedizin waren für die Organallokation in den Niederlanden wie auch in Großbritannien von großer Bedeutung.
Vorbereitende medizinische Maßnahmen

Nach der Entscheidung, die lebenserhaltende Behandlung abzubrechen, erfolgt eine vorläufige Beurteilung, ob der Patient als potenzieller Organspender infrage kommt. Die Niederländische Transplantationszentrale (Nederlandse Transplantatie Stichting, NTS) führt ein nationa- les Organspenderregister (https://www. donorregister.nl; [11]). Die Patientendaten werden überprüft auf eine:

a. registrierte Zustimmung zur Organspende,

b. registrierte Ablehnung zur Organspende,

c. Zustimmung oder Ablehnung durch Angehörige, 


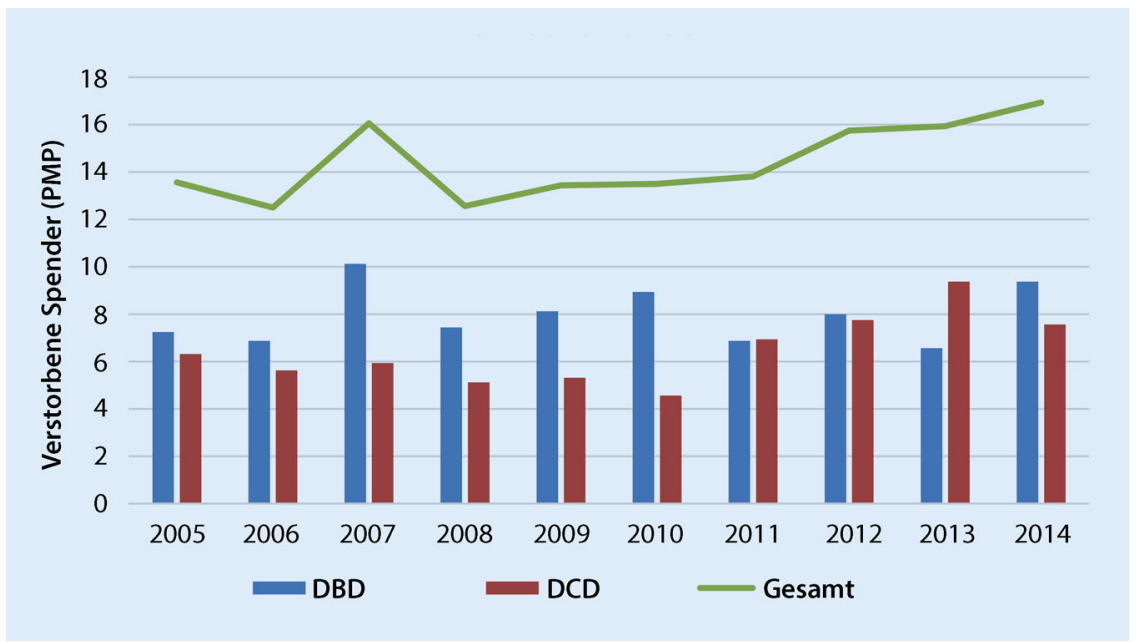

Abb. $3 \triangle$ Anzahl der Organspender in den Niederlanden pro 1 Mio. Einwohner (PMP) in den vergangenen 10 Jahren. Blaue Säule DBD (Donation after Brain Death = heart-beating donation, Organspende nach Hirntod = Organspende bei Herzschlag), Rote Säule DCD (Donation after Circulatory Death = non-heart-beating donation, Organspende nach Herz-Kreislauf-Tod=Organspende ohne Herzschlag)

d. Zustimmung oder Ablehnung durch genau benannte Person,

e. keine Anmeldung im Register.

Einträge im niederländischen Spenderregister können jederzeit eingesehen werden. Bei Nichtregistrierung wird der mutmaßliche letzte Wille des Patienten mit den nächsten Angehörigen besprochen, die dann einer Organspende zustimmen oder diese verneinen können. Bei Ablehnung zur Organspende wird das Allokationsverfahren beendet und der Patient wird, entsprechend den „End-of-life-care“-Richtlinien, palliativ behandelt. Im Falle einer Zustimmung durch den Patienten oder durch Angehörige/Betreuer wird der Ablauf eines Organspendeverfahrens mit der Familie besprochen. Gleichzeitig werden die Patientendaten der Niederländischen Transplantationszentrale übermittelt.

Der Prozess der Spende nach dem Hirntod (DBD) unterscheidet sich von dem Prozess der Spende nach dem HerzKreislauf-Tod (DCD). Bei DBD muss die Hirntoddiagnostik abgeschlossen und der Tod festgestellt sein, anschließend kann die Explantation geplant und durchgeführt werden. Die Herausforderung bei der DCD besteht darin, die Prozesse des Entscheids zum Therapieabbruch und zur Organentnahme zu trennen. Hier kommt es zur Aufgabenteilung zwischen dem Behandlerteam der Intensivstation, dem hinzugezogenen Transplantationskoordinator (TC) und dem Explantationsteam [11].

Vorbereitende medizinische Maßnahmen, wie z. B. künstliche Beatmung, Volumentherapie oder der Einsatz vasoaktiver Medikamente zur Verbesserung der Hämodynamik, werden bei potenziellen Organspendern zwischen dem Therapieabbruch und der Organentnahme durchgeführt. Sie sind nötig, um die Organe vor Schaden zu bewahren. In der letzten Überarbeitung des niederländischen Transplantationsgesetzes wurde der Rechtsstatus zu vorbereitenden medizinischen Maßnahmen bei potenziellen Organspendern festgelegt [11].

\section{Zentrale Rolle des \\ Transplantationskoordinators}

Der Transplantationskoordinator (TC) kommt in die entsprechende Klinik des Spenders, koordiniert das Organspendenverfahren, informiert und betreut die Angehörigen, beurteilt in Kooperation mit Fachärzten die Qualität der Organe, erstellt einen Zeitplan und übermittelt alle wichtigen Daten der Europäischen Vermittlungsstelle für Organtransplantationen. Die Stiftung Eurotransplant ist als Serviceorganisation verantwortlich für die Zuteilung von Spenderorganen in acht europäischen Ländern [11].

\section{Autonome Explantationsteams}

Die Organspende wird dann von einem externen und völlig autonomen Explantationsteam (Zelfstandig Uitname Team, ZUT) durchgeführt. In den Niederlanden wurden hierzu 5 Teams eingerichtet (Region West: durch Teams der Universitätskliniken Rotterdam und Leiden, Region Ost: durch Teams der Universitätskliniken Groningen, Nijmegen und Maastricht), sodass jederzeit eine Explantation unabhängig vom eigenen Krankenhauspersonal durchgeführt werden kann. Das ZUT besteht aus zwei Chirurgen, zwei Operationsassistenten, einem Anästhesisten und einer Anästhesiepflegekraft. Um das "Spenderkrankenhaus" zu entlasten, wird die gesamte chirurgische und anästhesiologische Ausrüstung inklusive der Operationsbekleidung und des Verbandmaterials mitgenommen. Benötigt wird lediglich ein freier Operationssaal und ein Narkosegerät. Diese Maßnahmen reduzierten erheblich die Vorbereitungszeit zur Organspende [11].

\section{Feststellung des Todes nach Herz-Kreislauf-Stillstand}

Der Zeitfaktor ist bei einer DCD ein sehr wichtiges Element. Zum einen musste nach dem Herzstillstand genügend Zeit verstrichen sein, damit der irreversible Funktionsausfall des Gehirns aufgrund der fehlenden Durchblutung gesichert festgestellt werden kann und das Todeskriterium erfüllt ist („dead donor rule“). Zum anderen musste hinsichtlich der Erhaltung der Organqualität die Zeit zwischen der Todesfeststellung und der Organentnahme so kurz wie möglich sein. Die Organvermittlungszentrale Eurotransplantat erklärte 1995, dass ein Herz-Kreislauf-Stillstand von $10 \mathrm{~min}$ gleichwertig zum Hirntod sei [14].

Der Zeitpunkt des Herz-KreislaufTodes wurde anfänglich mittels EKG überwacht und festgestellt. Zwischen dem Eintreten des Kreislaufstillstands und dem Beenden der elektrischen Aktivität wurden bei jungen Organspendern jedoch Zeitspannen bis zu 30 min gemessen. Damit waren die meisten Spenderorgane nicht mehr transplantierbar. 


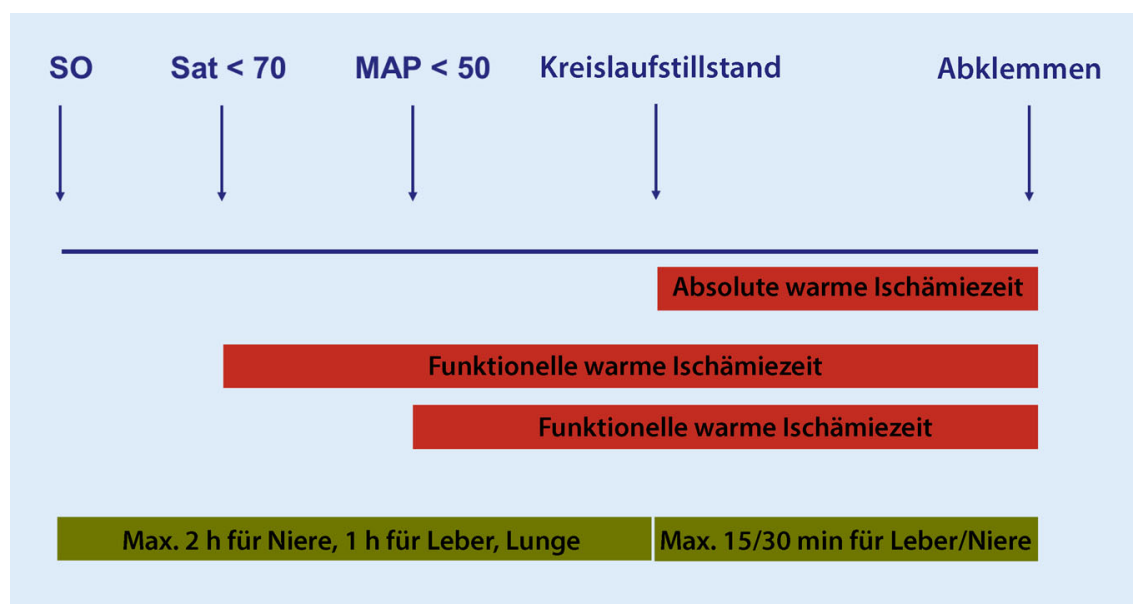

Abb. 4 ム Zeitliche Abfolge einer "donation after circulatory arrest" (DCD). Die maximale Dauer zwischen dem "switch off" und dem Kreislaufstillstand ist in den Niederlanden für Nieren auf maximal $2 \mathrm{~h}$ und für Leber und Lunge auf $1 \mathrm{~h}$ beschränkt. In Großbritannien beträgt die maximale Dauer sogar $3 \mathrm{~h}$. Zur Berechnung der funktionellen warmen Ischämiezeit wird der Eintritt der Hypoxie und/oder der arteriellen Hypotension miteinbezogen. Die absolute warme Ischämiezeit beginnt mit dem Eintritt des Kreislaufstillstandes. MAP "mean arterial blood pressure", Sat „oxygen saturation ", SO "switch off" (Beenden der lebenserhaltenden Therapie)

Nationale und internationale Arbeitsgruppen verschiedener medizinischer Fachgesellschaften publizieren hierzu Standpunkte und Empfehlungen [22, 35].

In den Niederlanden wurden mittlerweile bindende Leitlinien verfasst [11].

Die Feststellung des Todes basiert nun auf dem Sistieren des Blutstroms.

Hierzu wird dem Organspender ein arterieller Zugang gelegt. Wenn keine Druckwelle im arteriellen System mehr nachgewiesen werden kann, ist das Kriterium des Herz-Kreislauf-Stillstandes erfüllt. Nach einer 5-minütigen Observationszeit („no-touch time“) wird der Tod diagnostiziert. Hier werden dieselben klinischen Untersuchungen durchgeführt, die auch als Todesnachweis für Hirntod notwendig sind (beidseits weite, nicht auf Licht reagierende Pupillen, keine Hirnreaktion auf schmerzhafte Reize; fehlender Husten- und Schluckreflex, keine Spontanatmung). Die klinischen Zeichen des Todes müssen gemeinsam von zwei Ärztinnen oder Ärzten mit entsprechender fachlicher Qualifikation diagnostiziert werden (Vier-AugenPrinzip).

Auch im Sprachgebrauch der postmortalen Organspende findet sich eine
Organisation des "switch off" und Umgang mit den Angehörigen des Organspenders

Die Angehörigen müssen darüber informiert werden, dass der zum Tod führende Kreislaufstillstand in den meisten Fällen rasch nach Abbruch der Therapie (z.B. künstliche Beatmung, Einsatz von Vasopressoren und Inotropika) eintritt, dass es aber auch mehrere Stunden bis zum Eintreten des Todes dauern kann. In letzterem Fall ist eine Organentnahme aus medizinischen Gründen nicht mehr möglich. Zudem müssen die Angehörigen verstehen, dass nach Eintreten des Todes ein hoher Zeitdruck besteht, die Organe zu entnehmen.

Bei der Planung und Logistik des Abbruchs der lebenserhaltenden Maßnahmen („switch off“) werden die Bedürfnisse und Wünsche der Angehörigen miteinbezogen. Die Angehörigen dürfen beim „switch off“ anwesend sein. Sie werden sowohl durch den behandelnden Arzt als auch durch den TC begleitet. Die Verantwortung des „switch off“ obliegt dem medizinischen Behandlerteam der Intensivstation. Der Ablauf des „switch off“, Wartezeiten, Feststellung des Todes, Verbleib des Leichnams, Verbleib der Angehörigen müssen deutlich besprochen sein.

Vor der Explantation führen der TC und das ZUT eine „time-out procedure" durch. Hierbei werden, gemäß einer Checkliste, Identität des Spenders, Krankengeschichte, Organspendeprotokolle und der aktuelle Status des Spenders besprochen und überprüft. Nach der Feststellung des Todes, nimmt die Familie Abschied und der Verstorbene wird unmittelbar zur Organspende in den Operationssaal gebracht. Im Operationssaal werden Identität und Kreislaufstillstandes des Organspenders erneut überprüft. Nach Vorbereitung erfolgt die Notlaparotomie. Die Aorta abdominalis, Aorta ascendens, Arteria pulmonalis, Vena cava inferior und superior sowie das linke Herzohr werden freigelegt. Nach Abklemmen der Aorta abdominalis oberhalb des Truncus coeliacus erfolgt eine Druckperfusion der Organe mit kalter Konservierungslösung über die Aortenwurzel. Die Schritte der DCD 
sind in - Abb. 4 dargestellt. Die Explantation dauert etwa 3-6 h. Der Leichnam wird nach der Explantation auf die Intensivstation und anschließend in die Leichenhalle gebracht.

Die Haltung der Angehörigen zur DCD ist im Allgemeinen negativer verglichen mit der DBD. Unserer Erfahrung nach begründet sich dies durch den erhöhten Zeitdruck innerhalb einer DCD und den dadurch verminderten Puffer für die seelsorgerische Betreuung der Angehörigen. In dieser Situation ist es für das intensivmedizinische Behandelteam sehr wichtig eine klare Aufgabenteilung hinsichtlich der Pflege des Organspenders und der Angehörigenbetreuung zu verabreden. Die Anwesenheit der Familie beim „switch off“ und das standardisierte Vorgehen aller Beteiligten hat die Akzeptanz der DCD deutlich verbessert.

In ungefähr 20\% der DCD-Fälle verzögert sich die Zeitdauer zwischen „switch off“ und Kreislaufstillstand. Nach Überscheiten der Fristzeiten (1 h für Leber und Lungen; 2 h für Nierenspende) ist eine Transplantation dieser Organe nicht mehr sinnvoll und die DCD wird abgesagt.

\section{Das Phänomen der Autoreanimation}

Nach erfolgloser Reanimation kann es wenige Minuten später zu einer plötzlichen Rückkehr der spontanen Kreislauffunktion kommen. Das Phänomen der Autoreanimation (,spontaneus return of circulation“, SROC), auch als „LazarusPhänomen “ benannt, ist in der medizinischen Fachliteratur eher selten beschrieben. Trotzdem tritt es (auch bei Berücksichtigung berichteter, aber medizinisch nicht publizierter Fälle) häufiger auf als angenommen. Der längste beobachtete Zeitraum zwischen Asystolie und Autoreanimation betrug $7 \mathrm{~min}$ nach Beenden der Reanimationsmaßnahmen [13].

Auch in der "no-touch time “ bei DCD wurde das Phänomen der Autoreanimation in einigen Fällen beschrieben [8]. Dies führte in keinem der Fälle zu einer klinisch nachweisbaren Wiederherstellung von Lebenszeichen oder Überleben. In einer retrospektiven Übersichtsarbeit wurden 73 Fälle einer eDCD analysiert. Die durchschnittliche Zeitdauer zwischen dem "switch off“ und dem Kreislaufstillstand betrug $22 \mathrm{~min}$. Bei keinen dieser Patienten trat in den ersten 5 min nach Einsetzen der Asystolie eine Autoreanimation auf [27]. Bei der erwarteten DCD, in der keine Reanimation durchgeführt wird, erscheint eine „notouch time“ von 5 min daher ausreichend. Bei der unerwarteten DCD nach erfolgloser Reanimation hingegen ist eine 5-minütige Wartezeit wahrscheinlich unzureichend.

Nach 15 Jahren Erfahrung mit eDCD in den Niederlanden wurden glücklicherweise nur sehr seltene Fälle von Autoreanimation dokumentiert, jedoch nicht publiziert. Das Auftreten einer Autoreanimation stellt sowohl für die für die Angehörigen als auch für das intensivmedizinische Personal eine schwere medizinische und emotionale Belastung dar. Deshalb sollten das Phänomen der Autoreanimation und deren Auswirkungen zwischen den Angehörigen und dem intensivmedizinischen Behandlerteam vorab besprochen werden. Schriftliche Handlungsanweisungen helfen, auf diese Form eines Zwischenfalls transparent und kompetent reagieren zu können.

Bei Auftreten einer Autoreanimation bei eDCD empfehlen wir, die Angehörigen zu beruhigen, ihnen das Phänomen erneut zu erklären und nicht zu reanimieren, da es ja für den Patienten keine kurative Behandlung gibt. Die eDCD wird passager unterbrochen. Nach erneutem Kreislaufstillstand muss eine 5-minütige „no-touch time“ abgewartet werden, danach wird die eDCD fortgesetzt. Sollten die Pufferzeiten für eine Organspende überschritten sein, verstirbt der Patient ohne Organspende auf der Intensivstation.

Wissenschaftliche Erklärungen bezüglich der Ursachen einer Autoreanimation können nicht evidenzbasiert begründet werden. Als Ursachen werden "autopositive“ endexspiratorische Drücke, Hyperventilation mit reaktiver Alkalose, Intubationsreiz, Hyperkaliämie, verzögerte Medikamentenwirkung und nicht beobachtete „vita minima" diskutiert. Weitere wissenschaftliche experimentelle Arbeiten erscheinen not- wendig, um den Pathomechanismus dieses Phänomens besser zu verstehen. Im Falle einer Organspende mit Lungenexplantation empfehlen wir, zur Vermeidung hoher Beatmungsdrücke, erst nach der Kanülierung und Perfusion den Spender endotracheal zu intubieren und ventilieren.

\section{Schlussfolgerungen}

In den Niederlanden leben ca. 17 Mio. Einwohner. Die Anzahl an potenziellen Organspendern war mit 10 Transplantationen pro 1 Mio. Einwohner die niedrigste in ganz Europa. Viele Patienten auf der Warteliste verstarben vor der Transplantation. Die Transplantationsmedizin stand in den Niederlanden vor großen Herausforderungen. Medizinische Fachgesellschaften, öffentliches Gesundheitswesen, Krankenkassen und Gesetzgeber mussten Wege zu einer verbesserten Organallokation, Logistik und Anzahl von Transplantationen finden. Viele nationale und lokale Projekte wurden im Rahmen zur Verbesserung des Organspendeprozesses durchgeführt. Viele Erfahrungen wurden durch Versuch und Irrtum gemacht.

\section{> Der wichtigste Schritt war die Einführung der DCD.}

Durch Maßnahmen wie z. B. die Einführung einer nationalen Organspenderdatenbank, eine verbesserte Aufklärung in der Öffentlichkeit, Weiterbildung auf den Intensivstationen, Leitlinien zur intensivmedizinischen Behandlung am Lebensende, Einsatz eines Transplantationskoordinators vor Ort, Einführung autonomer Explantationsteams und strikte Verfahrensanweisungen zum Ablauf der Organspende konnten viele praktische Fragen zu Logistik und Aufgabenteilung der DBD und DCD beantwortet werden.

Nach lang andauernder Debatte zu medizinisch-ethischen Themen der Organtransplantation konnten sich alle Beteiligten in verschieden Schritten auf ein gesellschaftlich-medizinisch-juristisches Regelwerk zu DCD und DBD einigen. Die Anzahl postmortaler Organspender stieg 2014 auf 16 pro 1 Mio. Einwohner. Mittlerweile stammen bis zu $60 \%$ der 
Organspenden in den Niederlanden in aus einem DCD-Verfahren, verglichen mit ungefähr $10 \%$ in den USA.

Das große Problem der postmortalen Organallokation bleibt trotz aller Verbesserungen bestehen. Die Entnahme von Organen ist von Staat zu Staat unterschiedlich geregelt. In Deutschland, Schweiz, Großbritannien, Dänemark und in den Niederlanden gilt die Regelung der erweiterten Zustimmung zur Organspende. Jeder Bürger kann aus eigener Initiative sein Einverständnis zur Organspende geben. Gibt es keine Festlegung, müssen die Angehörigen entscheiden. Bei der Widerspruchslösung wird dagegen von einem grundsätzlichen Einverständnis ausgegangen, es sei denn, der Betreffende hat zu Lebzeiten ausdrücklich widersprochen, wie z. B. in Österreich und Spanien. Kritik an dieser Lösung ist der Eingriff in die Persönlichkeitsrechte. Andere Staaten praktizieren die erweiterte Widerspruchslösung, bei der auch ein Widerspruch der Angehörigen gegen eine Organentnahme maßgebend ist (Finnland, Frankreich, Italien, Norwegen und Schweden). Länder mit „Widerspruchslösung“ verzeichnen häufig eine höhere Quote von Organspendern. In Spanien spenden 34, in Belgien 27 Menschen pro 1 Mio. Einwohner nach ihrem Tod mindestens ein Organ. Dieses Model könnte, sowohl für die Niederlande als auch für Deutschland, zu einer erheblichen Verbesserung der Organallokation führen.

\section{Fazit für die Praxis}

\section{- Die Bereitschaft zur Organspende muss nicht per se als Pflicht verstan- den werden. \\ - Die dahinter liegende Frage ist eine Wertfrage zur Gestaltung unseres Lebensendes. \\ - Hier müssen die Bürger und Bürgerin- nen mehr in die medizinisch-ethische Diskussion über Todesverständnis, Palliativmedizin, Patientenverfü- gung, Selbstbestimmung am Lebens- ende und die Frage der Organspende miteinbezogen werden.}

\section{Korrespondenzadresse}

Prof. Dr. MD PhD J. de Jonge

Department of Surgery, Erasmus University Medical Centre

Suite H813, 2040, 3000CA Rotterdam,

Netherlands

j.dejonge.1@erasmusmc.nl

\section{Einhaltung ethischer Richtlinien}

Interessenkonflikt. J. de Jonge, M. Kalisvaart, M. van der Hoeven, J. Epker, J. de Haan, J. N. M. IJzermans und F. Grüne geben an, dass kein Interessenkonflikt besteht.

Dieser Beitrag beinhaltet keine Studien an Menschen oder Tieren.

Open Access. This article is distributed under the terms of the Creative Commons Attribution 4.0 International License (http://creativecommons.org/ licenses/by/4.0/), which permits unrestricted use, distribution, and reproduction in any medium, provided you give appropriate credit to the original author(s) and the source, provide a link to the Creative Commons license, and indicate if changes were made.

\section{Literatur}

1. Abt P, Crawford M, Desai N et al (2003) Liver transplantation from controlled non-heartbeating donors: an increased incidence of biliary complications. Transplantation 75:1659-1663. doi:10.1097/01.TP.0000062574.18648.7C

2. Ad Hoc Committee of the Harvard Medical School (1968) A definition of irreversible coma. Report of the ad hoc committee of the harvard medical school to examine the definition of brain death. JAMA 205:337-340

3. Bernat JL, Capron AM, Bleck TP et al (2010) The circulatory-respiratory determination of death in organ donation. Crit Care Med 38:963-970. doi:10.1097/CCM.0b013e3181c58916

4. Deutscher Ethikrat (2015) Hirntod und Entscheidung zur Organspende. ISBN 978-3-941957-67-1, S1-189

5. EDQM (2015) International figures on organ, tissue \& hematopoietic stem cell donation \& transplantation activities. Documents produced by the Council of Europe, European Commitee (partial agreement) on organ transplantation (CDP-TO). Year 2014. NewsI Transpl 20:1-68

6. Eurotransplant International Foundation (2015) Statistics Report Library

7. Fenner H, Buss C, Gardiner D (2014) Intensive care staff attitudes to deceased organ donation. J Intensive Care Soc 15:53-56. doi:10.1177/175114371401500111

8. Gamboa E, Bronsther O, Halasz N (1986) The Lazarus phenomenon. Clin Transpl 1:125-127

9. Gerritsen RT, Kesecioglu J, Kompanje EJO et al (2009) Richtlijn nalaten en staken van behandeling en palliatieve zorg na het staken van behandeling bij volwassen IC patiënten (Richtlinie über das Beenden der lebenserhaltender intensivmedizinischer Maßnahmen und palliative Fürsorge bei Erwachsenen), S1-29

10. Gramm HJ, Zimmermann J, Meinhold $\mathrm{H}$ et al (1992) Hemodynamic responses to noxious stimuli in brain-dead organ donors. Intensive Care Med 18:493-495

11. Health Council of the Netherlands (2015) Determining death in postmortal organ donation Protocols and criteria, including an updated brain death protocol. Publication no. 2015/13.ISBN:97894-6281-040-2, S1-160

12. Hoffmann T, Minor T (2015) New strategies and concepts in organ preservation. Eur Surg Res 54:114-126. doi:10.1159/000369455

13. Hornby K, Hornby L, Shemie SD (2010) A systematic review of autoresuscitation after cardiac arrest. Crit Care Med 38:1246-1253. doi:10.1097/CCM.0b013e3181d8caaa

14. Kootstra G, Daemen JH, Oomen AP (1995) Categories of non-heart-beating donors. Transplant Proc 27:2893-2894

15. Manara AR, Murphy PG, O,Callaghan G (2012) Donation after circulatory death. Br J Anaesth 108(1):i108-i121.doi:10.1093/bja/aer357

16. Mindach M (2015) The German Ethics Council and the brain death - some clinical remarks. Fortschr Neurol Psychiatr 83:446-450. doi:10.1055/s-0035 1553544

17. Müller S (2011) Wie tot sind Hirn-tote? Alte Frageneue Antworten. PolitZeitgesch 20-21:3-9

18. Nederlandse Transplantatie Stichting (2015) NTS Jaarverslag 2014, S1-120

19. Nederlandse Transplantatie Stichtung (NTS), Nederlandse Orgaantransplantatie Registratie (NOTR), http://www.transplantatiestichting.nl/ cijfers/overlevingscurves

20. President's Council on Bioethics (2008) Controversies in the determinations of death, S1-168

21. Renz JF (2008) Is DCD for liver transplantation DNR? Am J Transplant 8:485-488. doi:10.1111/j.16006143.2007.02111.x

22. Rudge C, Matesanz R, Delmonico FL, Chapman J (2012) International practices of organ donation. $\mathrm{Br}$ J Anaesth 108(1):i48-i55. doi:10.1093/bja/aer399

23. Schaetzler TG (2015) Transplantations-Paradigma? Leserbrief (25.02.2015) zu: Ethikrat plädiert für Hirntod als Kriterium für die Organentnahme. Dtsch Arztebl (2015) Nachricht Nr. 61921 (24.02.2015), http://www.aerzteblatt. de/nachrichten/61921/Ethikrat-plaediert-fuerHirntod-als-Kriterium-fuer-die-Organentnahme

24. Shemie SD (2014) Life, death, and the bridges in-between. Ann NY Acad Sci 1330:101-104. doi:10.1111/nyas.12564

25. Shemie SD, Baker A (2014) Where have we been? Where are we going? Initiatives to improve uniformity of policies, integrity of practice, and improve understanding of brain death within the global medical community and lay public. J Crit Care 29:1114-1116. doi:10.1016/j.jcrc.2014.08.007

26. Shemie SD, Hornby L, Baker A et al (2014) International guideline development for the determination of death. Intensive Care Med 40:788-797. doi:10.1007/s00134-014-3242-7

27. Sheth KN, Nutter T, Stein DM et al (2012) Autoresuscitation after asystole in patients being considered for organ donation. Crit Care Med 40:158-161. doi:10.1097/CCM.0b013e31822f0b2a

28. Skaro Al, Jay CL, Baker TB et al (2009) The impact of ischemic cholangiopathy in liver transplantation using donors after cardiac death: the untold story. Surgery 146:543-552 (discussion 552-553) doi:10.1016/j.surg.2009.06.052

29. Summers DM, Johnson RJ, Allen J et al (2010) Analysis of factors that affect outcome after transplantation of kidneys donated after cardiac death in the UK: a cohort study. Lancet 376:1303-1311. doi:10.1016/S0140-6736(10)60827-6 
30. Truog RD (2007) Brain death - too flawed to endure, too ingrained to abandon. J Law Med Ethics 35:273-281. doi:10.1111/j.1748720X.2007.00136.x

31. Truog RD, Miller FG (2008) The dead donor rule and organ transplantation. N Engl J Med 359:674-675. doi:10.1056/NEJMp0804474

32. Truog RD, Miller FG, Halpern SD (2013) The deaddonor rule and the future of organ donation. N Engl J Med 369:1287-1289. doi:10.1056/ NEJMp1307220

33. Weber M, Dindo D, Demartines N et al (2002) Kidney transplantation from donors without a heartbeat. NEngl J Med 347:248-255. doi:10.1056/ NEJMoa020274

34. White SA, Prasad KR (2006) Liver transplantation from non-heart beating donors. BMJ 332:376-377. doi:10.1136/bmj.332.7538.376

35. Wijdicks EFM, Varelas PN, Gronseth GS et al (2010) Evidence-based guideline update: determining brain death in adults: report of the Quality Standards Subcommittee of the American Academy of Neurology. Neurology 74:1911-1918. doi:10.1212/WNL.0b013e3181e242a8

36. Young PJ, Matta BF (2000) Anaesthesia for organ donation in the brainstem dead - why bother? Anaesthesia 55:105-106

37. Sánchez-Fructuoso Al, Prats D, Torrente J, PérezContín MJ, Fernández C, Alvarez J et al (2000) Renal transplantation from non-heart beating donors: a promising alternative to enlarge the donor pool. J Am Soc Nephrol 11:350-358. http://jasn. asnjournals.org/content/11/2/350.long

38. Eurotransplant International Foundation (2015) Statistics Report Library. Chart - deceased donors used, per million population, by year, by donor country. Datei: 1036P_all organs. http://statistics. eurotransplant.org. Zügegriffen: 8.12.2015

\section{Viele Ärzte sind Vorsorgemuffel}

Viele Ärzte neigen offenbar dazu, ihre eigene Gesundheit zu vernachlässigen. Unter diesem Gesichtspunkt haben nun chinesische Forscher aus Taiwan zwei nationale Krebsdatenbanken nach den sechs häufigsten Tumordiagnosen auf der Insel durchforstet. Dazu zählen Wucherungen in Lunge, Darm, Leber, Mund- und Speiseröhre sowie in der weiblichen Brust und im Gebärmutterhals. An solchen Tumoren erkrankten zwischen den Jahren 1999 und 2012 insgesamt 542 Ärzte. Jedem der Ärzte stellten sie nun fünf Nichtmediziner mit gleichem Geschlecht, Alter und Wohnort und ähnlichem Einkommen gegenüber (propensity score matching).

\section{Ähnliche Krebsstadien bei Diagnose}

Wie sich zeigte, gab es in der Summe keine großen Unterschiede bei den Krebsstadien zum Zeitpunkt der Diagnose: Knapp neun Prozent der Ärzte hatten einen Stadium-0-Tumor, die übrigen Ärzte verteilten sich fast gleichmäßig auf die anderen Stadien; signifikante Unterschiede zu Nichtmedizinern wurden dabei nicht beobachtet. Stadium-IV-Tumoren kamen bei Ärzten zwar tendenziell etwas häufiger vor, der Unterschied zu Nicht-Medizinern war allerdings nicht signifikant. Auch eine Stratifizierung nach Wohnort, Geschlecht oder Einkommen der Studienteilnehmer ergab keine wesentlichen Differenzen. Ärzte in sehr ländlichen Gebieten scheinen bei der Diagnose aber seltener einen Stadium-IV-Tumor zu haben als der Rest der dortigen Bevölkerung (16 versus 25 Prozent), allerdings war auch dieser Unterschied statistisch nicht signifikant. Deutliche Unterschiede fanden die Gesundheitsforscher lediglich bei Frauen-bezogenen Tumoren. So stellten sie bei Ärztinnen zum Diagnosezeitpunkt etwa zweieinhalbfach häufiger ein metastasiertes Brust- oder Zervixkarzinom fest als bei Frauen ohne Approbation.

\section{Ärztinnen nutzen Vorsorge selten}

Zwar gibt es in Taiwan seit dem Jahre 1995 ein Screeningprogramm auf Zervixkarzinome, dies wird offenkundig von Ärztinnen selbst aber kaum in Anspruch genommen. Nach Umfragen nehmen außerdem nur rund zwölf Prozent des weiblichen Medizinpersonals an Mammografie-Untersuchungen teil, nur jede Zehnte geht regelmäßig zu den wichtigsten Krebsvorsorgeuntersuchungen, berichten die taiwanesischen Wissenschaftler. Auch in anderen Ländern sind Ärzte häufig Vorsorgemuffel. So haben Umfragen ergeben, dass zwar 60 Prozent der israelischen Hausärzte die Vorsorge empfehlen, aber nur jeder vierte selbst daran teilnimmt. Und Studien in Kanada haben gezeigt, dass 40 Prozent der Ärzte und Ärztinnen in den vergangenen fünf Jahren keine Prostata- beziehungsweise Brustkrebsuntersuchung bei sich selbst haben machen lassen, wie die chinesischen Forscher berichten. Über die Gründe für dieses Verhalten lässt sich nur spekulieren: Möglicherweise ist die hohe Arbeitsbelastung bei Ärzten eine Ursache für solche Nachlässigkeiten. Allerdings wäre dann zu erwarten, dass auch andere Berufsgruppen mit einem vergleichbaren Einkommen kaum Zeit für die Vorsorge finden.

\section{Quelle:Ärztezeitung, www.aerztezeitung.de}

basierend auf: CMAJ (2015) online 20. Juli 